\title{
Morphological changes induced by extensive endobronchial electrocautery
}

\author{
C. Verkindre*, A. Brichet*, C.A. Maurage**, P. Ramon*, J-P. Homasson ${ }^{+}$, C.H. Marquette*
}

\begin{abstract}
Morphological changes induced by extensive endobronchial electrocautery. C. Verkindre, A. Brichet, C.A. Maurage, P. Ramon, J-P. Homasson, C.H. Marquette. (C)ERS Journals Ltd 1999.
\end{abstract}

ABSTRACT: Due to recent improvements of safety conditions for therapeutic devices, electrocautery is being considered with renewed interest in the field of therapeutic bronchoscopy. The efficiency of this technique for destructing intraluminal tumours is well documented and makes it an attractive alternative to Yttrium aluminium garnet (YAG) laser photo-coagulation. Little is known, however, about the morphologic changes induced by electrocautery within the bronchial wall structures. This information is, however, important since electrocautery has been proposed as an alternative to other techniques to treat superficial tumours of the bronchial wall.

Soft coagulation, with autostop, using two different power setting (40 and $120 \mathrm{~W})$, produced by a new generation of high frequency voltage regulated generators was applied circumferentially to the trachea or left main bronchus, in a series of 52 piglets. Early (48 h) and late effects (6 weeks) were assessed through gross examination (bronchoscopy and autopsy) and light microscopy.

Early effects of electrocautery included coagulation necrosis of the mucosa only and intense acute inflammation extending deep into the bronchial structure. The inflammatory phase progressively resolved while extensive transmural fibrosis and deterioration of the cartilage plates developed. The nature and extent of these lesions did not depend upon the energy delivered $(40 \mathrm{~W}$ versus $120 \mathrm{~W})$. Retractile scar formation and loss of cartilaginous support then produced iatrogenic secondary stenoses.

These results do not question the use of electrocautery to palliate endoluminal tumours but should make operators careful when treating extensive infiltration of the bronchial wall.

Eur Respir J 1999; 14: 796-799.
*Clinique des Maladies Respiratoires, Hôpital A. Calmette, CHRU de Lille, France; **Service d'Anatomopathologie, Hôpital A. Calmette, CHRU de Lille, France; ${ }^{+}$Centre Hospitalier Spécialisé en Pneumologie, Chevilly-Larue, France.

Correspondence: C.H. Marquette

Clinique des Maladies Respiratoires

Hôpital A. Calmette

CHU de Lille

59037 Lille cedex

France

Fax: 33320445177

Keywords: Electrocautery

therapeutic bronchoscopy

Received: September 101998

Accepted after revision May 151999

This work was supported by the Comité National contre les Maladies Respiratoires et la Tuberculose and by the friends and family of Michel Dubuisson ${ }^{\dagger}$.
Malignant tracheobronchial obstruction can cause major respiratory problems including dyspnoea, haemoptysis, postobstructive pneumonia and sometimes death due to terminal asphyxia.

Relief of obstruction of central airways can be achieved by several palliative techniques. External compression of the airways can be palliated with stent insertion. The intraluminal component of the tumour can be destroyed through Yttrium aluminium garnet (YAG) laser photocoagulation or electrocautery or with a delayed effect through cryotherapy, brachytherapy and photodynamic therapy.

Electrocautery almost disappeared since the old generators were very dangerous, causing burns, perforation and massive haemoptysis.

A renewed interest in the technique appeared as a result of the availability of new and safe generators of high frequency (HF) current, new probes easily usable through rigid and flexible bronchoscopes and, more recently, new insulated and electrically grounded flexible bronchoscopes [1-7].

As for Nd-YAG laser photocoagulation, electrosurgical tumour destruction is an immediate effect of thermal damage. For electrocautery the thermal effect is due to the passage of electric flows through the tissue. The depth of tissue necrosis depends on the voltage difference between the probe and the tissue and on the duration of contact. Even with the very new voltage regulated generators the depth of the necrosis which will be obtained can hardly be predicted. In addition, little is known about the morphologic changes induced by electrocautery on the normal bronchial structures. These unresolved issues clearly limit the use of the technique and could be at least partly resolved by experimental studies [2, 8, 9]. This is especially important since electrocautery has recently been proposed as an alternative to cryotherapy, brachytherapy or photodynamic therapy to treat superficial tumours of the bronchial wall [7].

The questions of this study were: does the safest mode of coagulation (i.e. "soft coagulation" mode with autostop) entail a risk of secondary stenosis? Does the power setting (40 versus $120 \mathrm{~W}$ ) influence the risk of secondary stenosis? What is the pathological substratum for these secondary stenoses?

The authors therefore evaluated the immediate and late effects of electrocautery delivered through rigid bronchoscopy to the normal tracheobronchial tree in piglets. Of particular interest was the study of acute and late changes of the mucosa, the submucosa and the cartilage. 


\section{Materials and methods}

\section{Animals}

Piglets (Largewhite-Landrace) weighing $23 \pm 6 \mathrm{~kg}$ were used. All animals were treated in compliance with the guidelines of the Department of Experimental Research of the Lille University and with the "Guide for the Care and Use of Laboratory Animals" [10]. In addition, the experimental protocols were reviewed and approved by the Animal Experimental Committee of the French ministry of agriculture.

Pigs of an average weight of $23 \mathrm{~kg}$ were chosen because their tracheal size approximates that of a human adult weighing 45-60 kg. This allows rigid bronchoscopy with the same instruments as those used in humans, and thus facilitates further extrapolation of the experience gained with therapeutic bronchoscopy in the pig models of central airway diseases [11].

\section{Bronchoscopic procedures}

All of the bronchoscopies were performed under general anaesthesia. The animals were premedicated intramuscularly with ketamine $(50 \mathrm{mg})$ and atropine sulphate $(0.25$ $\mathrm{mg})$. Anesthesia was induced with intravenous propofol $\left(2-3 \mathrm{mg} \cdot \mathrm{kg}\right.$ body weight $\left.{ }^{-1}\right)$ followed by a continuous infusion of propofol $\left(10 \mathrm{mg} \cdot \mathrm{kg}\right.$ body weight $\left.{ }^{-1}\right)$ for maintenance. Analgesia was provided by repeated intravenous bolus doses of $0.5 \mathrm{mg}$ alfentanyl. Neuromuscular blockade was obtained with repeated intravenous bolus doses of suxametonium $\left(0.1 \mathrm{mg} \cdot \mathrm{kg}\right.$ body weight $\left.{ }^{-1}\right)$. An $11 \mathrm{~mm}$ outer diameter rigid bronchoscope (Shapsay bronchoscope ref 10317 LA; Karl Storz Gmbh, Tuttlingen, Germany) with a $5.5 \mathrm{~mm}$ rigid telescope (Hopkins ref 10320 A; Karl Storz $\mathrm{GmbH}$ ) was used for bronchoscopy. Ventilation was provided by a high frequency jet ventilator through a canula adjusted to the proximal port of the rigid bronchoscope. Blood pressure, pulse oxymetry and electrocardiogram were monitored throughout the procedures.

\section{Electrocautery}

Electrocoagulation was uniformly applied circumferentially to a $2 \mathrm{~cm}$ long segment of the mid trachea (T) or to a $1 \mathrm{~cm}$ long segment of the left main stem bronchus (LB) with a semi rigid $2.5 \mathrm{~mm}$ diameter monopolar electrode (ref 20191-156; Erbe Elektromedizin Gmbh, Tübingen, Germany). Electric power was supplied by an HF generator (Erbotom ICC 350; Erbe Elektromedizin Gmbh) which was set on the "soft coagulation" mode with autostop. With these parameters there is no risk of electric arcs as with the "forced" or "spray" coagulation modes. The automatic voltage control present in the Erbotom ICC 350 (Erbe Elektromedirin) also ensures a high reproducibility of the soft coagulation depth. Finally, when setting the generator on "auto-stop", the power switches off as soon as vapour emerges from the coagulation zone, preventing adhesion of the probe to the tissues. Two levels of energy were studied $40 \mathrm{~W}$ and $120 \mathrm{~W}$ respectively. Thus, four groups of thirteen pigs were studied according to the site and the energy delivered: T $40 \mathrm{~W}, \mathrm{~T} 120 \mathrm{~W}, \mathrm{LB} 40 \mathrm{~W}$ and LB $120 \mathrm{~W}$.
In each group the early effects of electrocoagulation were assessed in 5 pigs, at $48 \mathrm{~h}$, through bronchoscopic and histopathologic examination of the coagulated area. The late effects were assessed in the remaining 8 pigs from each group after 6 weeks.

\section{Follow-up and evaluation}

Clinical signs were monitored daily with special attention to weight loss, cough, sputum production, wheezing and dyspnoea. Video recordings and photographs were taken on each bronchoscopic examination. Additional bronchoscopies were performed as warranted by clinical signs.

The animals were sacrificed at $48 \mathrm{~h}$ or 6 weeks postoperatively, depending on their study group, for post-mortem examination of the tracheobronchial tree. Euthanasia was performed under general anaesthesia with intravenous potassium chloride. Animals showing severe respiratory compromise before the predetermined time of sacrifice were euthanized under general anaesthesia.

Assessment of early and late effects of electrocoagulation was based on endoscopic gross findings and pathologic examination. Endoscopically, lesions at the level of the electrocoagulated zone were classified as "intrinsic" or "extrinsic" or "mixed" according to the respective presence of lesions within the lumen (pseudo membranes, granulomas), at the level of the bronchial wall (loss of cartilaginous support with subsequent collapse of bronchial wall) or both. Lungs, mediastinium, $\mathrm{T}$ and oesophagus were removed in one single block through a cervicothoracic midline incision for complete histopathological examination. The $\mathrm{T}$ and main stem bronchi were carefully excised and transverse and longitudinal sections were cut at the level of the electrocoagulated segments. Additional sections sampled above and under the zone of interest served as controls. Specimens were fixed in formalin for 3 weeks, embedded in paraffin, cut into $4 \mu \mathrm{m}$ slides and stained with haematoxylin-erythrosin-saffron (HES) for study with light microscopy.

\section{Results}

\section{Immediate effects}

Bronchoscopic procedures were all uneventful. Uniform whitish coagulation could easily be produced by successive circumferential applications of the electrode on $\sim 20$ adjacent sites. No fumes or unpleasant burnt flesh smell was observed. As commonly observed in clinical practice, with the preselected parameters (auto-stop and soft coagulation) the coagulation time was much shorter when delivering $120 \mathrm{~W}$ than when delivering $40 \mathrm{~W}$.

\section{Early effects}

No premature death was observed in the animals subjected to early $(48 \mathrm{~h})$ evaluation. Endoscopic examination revealed a whitish coagulation ring without gross ulceration, membranes, stenosis or perforation. Microscopic examination showed ulcerated mucosa covered by fibrinopurulent membranes or shed necrotic epithelium and acute inflammation of the mucosa, the submucosa and the perichondral spaces characterized by oedema and polymorphonuclear (sometimes altered) infiltrates. Acute vasculitis of the adventitial vessels was also a common finding. No changes were seen in the extracellular matrix 
of cartilage plates. The sites of coagulation (T versus LB) and energies delivered ( $40 \mathrm{~W}$ versus $120 \mathrm{~W}$ ) could not be distinguished on the basis of early gross and microscopic findings.

\section{Late effects}

All of the 8 pigs in the $\mathrm{T} 120 \mathrm{~W}$ group and $7 / 8$ pigs in the $\mathrm{T} 40 \mathrm{~W}$ group died prematurely, usually between the 10 th and the 20th days. In fact all of the premature deaths in these groups were related to euthanasia which was performed because the animals experienced severe respiratory failure. In the animals which received LB coagulation only five premature deaths were observed, one in the LB $120 \mathrm{~W}$ group and four in the LB $40 \mathrm{~W}$ group. These deaths occurred between the 20th and the 32nd days.

Endoscopic examination revealed severe stenosis $(\geq$ $80 \%$ ) in all the animals. In the $\mathrm{T} 120 \mathrm{~W}$ group the stenosis was classified as extrinsic in four animals and mixed in four. All the stenoses were classified as mixed in the T 40 $\mathrm{W}$ group. In the LB $120 \mathrm{~W}$ group the stenosis was classified as intrinsic in five animals, extrinsic in two and mixed in one. In the LB $40 \mathrm{~W}$ group the stenosis was classified as extrinsic in six and mixed in two. Most animals in the latter two groups presented postobstructive pneumonia on gross examination of the lungs. All the bronchial or tracheal stenoses classified as extrinsic or mixed had obvious loss of structural support on palpation.

Microscopic examination revealed that normal epithelium was replaced by squamous metaplasia in half of the cases. Acute inflammation persisted only at the level of the mucosa, except in those animals that died within the first 2 weeks, in which inflammatory lesions still extended deeply into the submucosal and perichondral spaces.

Mucosal fibrosis was a constant feature. This fibrosis always extended to the submucosa and to the cartilage plates. More than $50 \%$ of the normal basophilic extracellular cartilaginous matrix was replaced by saffron stained connective tissue (collagen). In these areas, there was an obvious loss of viable-appearing chondrocytes (figs. 1 and 2). No chronic lesions of the adventitial vessels were seen. As for early lesions, microscopic examination could not differentiate electrocoagulation produced with a power set at $40 \mathrm{~W}$ from that produced with a power set at $120 \mathrm{~W}$.

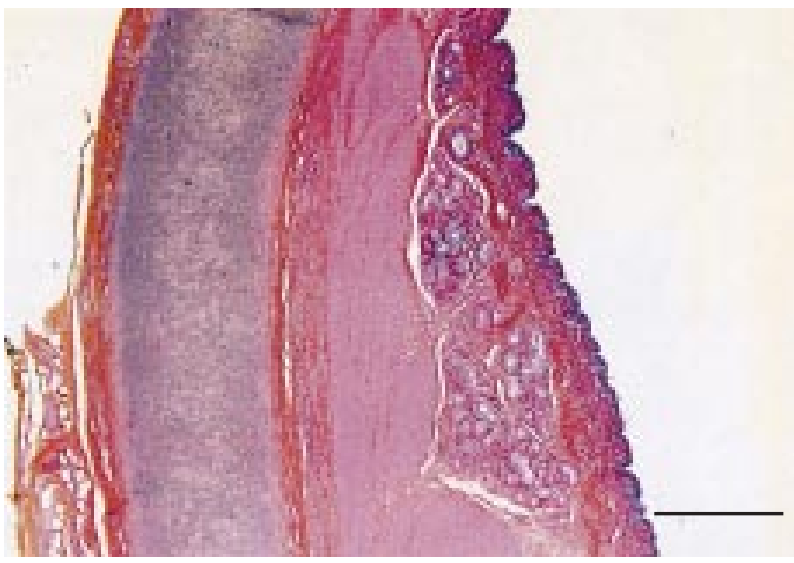

Fig. 1. - Normal bronchial section (haematoxylin-erythrosin stain), showing from right to left normal mucosa, submucosa and cartilaginous plate. Internal scale bar $=500 \mu \mathrm{m}$.

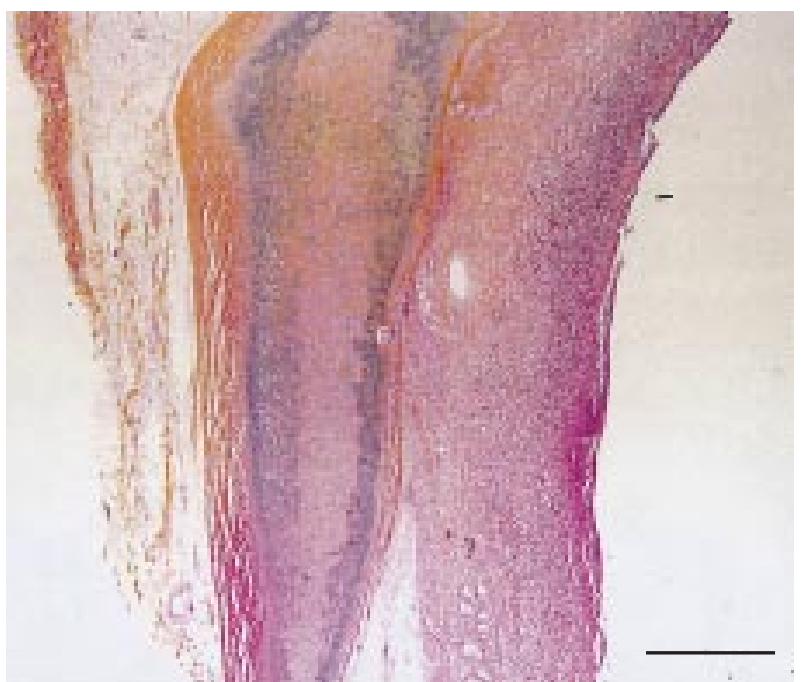

Fig. 2. - Bronchial section (haematoxylin-erythrosin-saffron stain) at the level at which electrocautery was applied 6 weeks before, showing shedding of the epithelium, intense submucosal fibrosis and degenerescence of the cartilaginous plate. Internal scale bar $=500 \mu \mathrm{m}$.

\section{Discussion}

The present study showed that even when using optimal safety parameters, extensive electrocoagulation of the bronchial mucosa may lead to serious structural damage deep into the tracheobronchial wall independently of the power setting. Mucosal and submucosal fibrosis and destruction of the cartilaginous support may in turn result in secondary iatrogenic stenoses. These lesions which occur after an intense and acute inflammatory phase do not result from extension of the coagulation necrosis into the bronchial wall

The safety of the new generation electrocautery equipment has improved in the recent past. The new HF generators have isolated outputs which reduce the risk of burns and electric shock to the endoscopist and patient. Insulated flexible bronchoscopes are now available. Automatic voltage control adjusts the thermal energy delivered to the effective area of contact of the tissue and thus, at least in the soft coagulation mode enhances the reproducibility of coagulation depth. Soft coagulation is now preferred [6]. Indeed forced or spray coagulation or resection may result in firing, especially in the presence of high inspired oxygen concentration. Moreover, as shown by experimental studies, the resection mode may result in bronchial wall perforation [9]. With the "auto-stop" function the generators automatically switch off as soon as the boiling point of the tissue fluids has been reached. This prevents adhesion of the probe to the tissue and the unpleasant burnt flesh smell due to excessive tissue dehydration.

Although appropriate equipment and recent safety guidelines prevent severe immediate complication of electrocautery, little is known regarding the risk of delayed complications such as secondary stenoses. In an experimental study CARPENTER et al. [8], comparing endoscopic cryosurgery and electrocoagulation of bronchi in a series of 20 mongrel dogs reported this iatrogenic complication. With electrocoagulation it was shown that the extent and severity of the damage to bronchial structures were unpredictable and sometimes resulted in secondary stenoses. On the contrary, cryolesions were predictable and 
uniform, with constant preservation of the bronchial architecture and regeneration of normal mucosa. In this study the authors used an old generator without an automatic voltage control or the auto-stop function. Moreover the mode of coagulation was not explicated. As attested by the presence of dry, charred eschar at the site of electrocoagulation and haemorragic necrosis in the adjacent lung, it may be hypothesized that the profound structural lesions resulted from coagulation in excess. Secondary stenoses of segmental bronchi after endobronchial electrocautery were recently reported by the group of VAN BOXEM et al. [7]. In this study the authors used $\sim 25-45 \mathrm{~W}$ of energy to treat radiographically occult lung cancers. Electrocoagulation was applied bronchoscopically with a flexible probe onto the lesions with an additional marginal area of $0.5 \mathrm{~cm}$ of normal mucosa. No auto-stop system was used and power was delivered until coagulation became visible to the operator.

It therefore seemed important to verify whether with the current devices and techniques, the iatrogenic lesions recently reported by VAN BOXEM et al. [7] could be related to the profound ultrastructural damage obtained experimentally by CARPENTER et al. [8] with an old generator. The $\mathrm{HF}$ generator used in the present study provided optimal coagulation in terms of control and reproducibility of soft coagulation depth. The lower power used here was in accordance with common practice in humans. With the auto-stop function, increasing the energy delivered shortens the duration of coagulation (the boiling point is reached more quickly). One could therefore expect that, with a higher energy level the depth of the electrically induced lesions would be reduced. The high power (120 W) was tested in order to test this hypothesis. Preoperative and early postoperative bronchoscopic examination showed only minimal superficial whitish coagulation necrosis. Light microscopy, however, revealed that, although coagulation necrosis was limited to the mucosa, acute inflammation with massive infiltration of altered polymorphonuclear cells extended throughout the bronchial structures down to the adventitia. Postoperative mortality was high. As confirmed by autopsy and microscopic examination and in contrast with the observations of CARPENTER et al. [8] none of the deaths were due to bronchial wall perforation. The animals which received tracheal coagulation died as a result of severe tracheal stenosis and collapse. Those in which coagulation was applied on the LB died as a result of pneumonia developing below the LB stenosis. Bronchoscopy and autopsy showed frank alterations of the cartilaginous support which could be observed 2 weeks postoperatively. In contrast with the findings of CARPENTER et al. [8], the present study did not show extension of the mucosal necrosis into the depth of the bronchial wall. In fact, after an acute inflammatory phase, extensive transmural fibrosis and cartilaginous deterioration developed. Latrogenic stenosis thus resulted from retractile scar formation and loss of cartilaginous support. The nature and extend of these lesions did not depend upon the energy delivered (40 W versus $120 \mathrm{~W}$ ).

Electrocautery is becoming more and more popular in Europe. This is due to the low cost of the equipment and to the safety of the modern generators. In their centre the authors have electrocautery, YAG-laser, cryotherapy, brachytherapy and photodynamic-therapy at their disposal.
Among the 240 patients treated for central airway malignancies last year, electrocautery was used in $>60 \%$ of the cases and YAG-laser in $<5 \%$. In the opinion of the present authors, diaphragm stenoses are the only indications where YAG-laser are clearly superior to electrocautery, since YAG-laser allows very precise radial cutting of the diaphragm. These figures regarding the implemented use of electrocautery compared to YAG-laser are becoming true in many European centres which have both techniques at their disposal.

The results of the present study show that a potential risk to the bronchial wall do not question the use of electrocautery to palliate endoluminal tumours. In contrast, when treating superficial tumours of the bronchial wall, such as radiographically occult lung cancers or carcinomas in situ [7], the electrocautery probe can be applied to a large area of the bronchial wall circumference. From these results it can be inferred that, whatever the energy delivered, such extensive bronchial wall coagulation, even with the "safest" parameters, can produce severe ultrastructural damage with secondary stenosis formation. This risk of bronchial ultrastructure damage is also present with alternative treatments such as brachytherapy and photodynamic therapy but not with cryotherapy.

\section{References}

1. Hooper RG, Jackson FN. Endobronchial electrocautery. Chest 1985; 87: 712-714.

2. Wallace JM. Electrosurgery via the fiberoptic bronchoscope: a useful therapeutic technique? Chest 1985; 87: 705-706.

3. Hooper RG, Jackson FN. Endobronchial electrocautery. Chest 1988; 94: 595-598.

4. Sutedja TG, van Kralingen K, Schramel FM, Postmus PE. Fibreoptic bronchoscopic electrosurgery under local anaesthesia for rapid palliation in patients with central airway malignancies: a preliminary report. Thorax 1994; 49: $1243-1246$.

5. Homasson JP, Roden S, Angebault M, Phung TM, Ngugen PT, Phuong TN. Treatment of bronchial tumours with high-frequency thermocoagulation: preliminary studies. Rev Pneumol Clin 1995; 51: 77-81.

6. Homasson JP. Endobronchial electrocautery. Sem Respir Crit Care Med 1997; 18: 535-543.

7. van Boxem TJ, Venmans BJ, Schramel FM, et al. Radiographically occult lung cancer treated with fibreoptic bronchoscopic electrocautery: a pilot study of a simple and inexpensive technique. Eur Respir J 1998; 11: 169-172.

8. Carpenter RJ, Neel HB, Sanderson DR. Comparison of endoscopic cryosurgery and electrocoagulation of bronchi. Trans Am Acad Ophthalmol Otolaryngol 1977; 84: 313323.

9. Vorre P, Ilium P, Oster S, Reske-Nielsen E, Larsen KB. Morphology of tracheal scar after resection with $\mathrm{CO}_{2^{-}}$ laser and high frequency cutting loop. A study in normal pigs. Acta Otolaryngol (Stockh) 1989; 107: 307-312.

10. Guide for the Care and Use of Laboratory Animals. National Institutes of Health, Bethesda, MD, USA. Publication number 93-23, 1985.

11. Marquette Ch-H, Mensier E, Copin MC, et al. Experimental models of Tracheobronchial Stenoses: a useful tool for evaluating airway stents. Ann Thorac Surg 1995; 60: 651-656. 